\title{
Dependencia y adicción al teléfono inteligente en estudiantes universitarios
}

\author{
Julio J. Aldana-Zavala ${ }^{1}$, Patricio A. Vallejo Valdivieso ${ }^{2}$, Josía Jeseff Isea- Argüelles ${ }^{1}$, y Félix J. Colina-Ysea ${ }^{3,4}$ \\ (1) Red de Investigación Koinonia, Venezuela. (correo-e: julioaldanazavala@gmail.com; josiaisea@gmail.com) \\ (2) Universidad Técnica de Manabí-Ecuador. (correo-e: patricio_2871@yahoo.es) \\ (3) Universidad Científica del Sur, Lima, Perú. (correo-e: fcolina@cientifica.edu.pe) \\ (4) Universidad Tecnología del Perú, Lima, Perú. (correo-e: c19271@utp.edu.pe)
}

Recibido Mar. 4, 2021; Aceptado May. 5, 2021; Versión final Jun. 16, 2021, Publicado Oct. 2021

\section{Resumen}

El principal objetivo de este estudio es determinar la dependencia y adicción al teléfono inteligente en estudiantes universitarios a partir de la aplicación de la escala de dependencia y adicción al smartphone (EDAS). Se trabaja en función del método deductivo, mediante un tipo de investigación descriptiva con diseño de campo no experimental transversal. Se aplica un muestreo estratificado proporcional a una muestra poblacional conformada por 303 estudiantes universitarios. Se calculan medias para los tres componentes de EDAS. En el componente 1, la media general es de 3,87. Esto indica dependencia y adicción en la muestra estudiada. Los resultados muestran mayor adicción en la población femenina en comparación a la masculina. La mayor afección se encuentra relacionada a ansiedad, depresión, estrés, trastornos del sueño, como elementos generadores de dependencia y adicción al teléfono inteligente por parte de los estudiantes universitarios. Se concluye que existe dependencia y adicción al teléfono inteligente en estudiantes universitarios.

Palabras clave: salud mental; efectos psicológicos; adaptación; estudiante; medios electrónicos; teléfono inteligente

\section{Smartphone dependency and addiction in university students}

\begin{abstract}
The main objective of this study is to assess university student dependency and addiction to smartphones by applying a smartphone addiction scale (SAS). The methodology applied is deductive, descriptive, and uses a non-experimental cross-sectional field design. A proportional stratified sampling is applied to a sample of 303 university students. The means for the three SAS components are calculated. In component 1 , the general mean is equal to 3.87. This suggests that there is dependency and addiction in the sample surveyed. The results show greater addiction in females than in males. The most related conditions are anxiety, depression, stress, and sleep disorders. These elements generate student dependency and addiction to smartphones. It is concluded that there is dependency and addiction to smartphones by university students.
\end{abstract}




\section{INTRODUCCIÓN}

Como producto de la era digital, la adicción sin sustancias es un tema que progresivamente va tomando importancia en la literatura científica, esto se debe a que se relaciona con factores asociados a internet, redes sociales, y el uso de teléfonos inteligentes, como se recoge en el (DSM-5, APA, 2013, p. 316), con el nombre de "trastornos no relacionados con sustancias", codificada como "juego patológico 312.31 (F63.0)". Cabe acotar que, dentro de este segmento, no se encuentra totalmente clarificado el uso de teléfonos inteligentes, lo cual conlleva a visiones encontradas sobre si el uso prolongado del mismo debe ser considerado como "adicción o uso problemático" (Panova y Carbonell, 2018; Yu y Sussman, 2020). Por otro lado, estudios realizados por (Yu y Sussman, 2020; Demirci et al. 2015), confirman la presencia de ansiedad, depresión, estrés, problemas para conciliar el sueño, producto del uso excesivo al dispositivo móvil, especialmente, en estudiantes universitarios.

En este sentido, dado a la nueva normalidad social generada desde la aparición de la pandemia por SARSCoV-2, ha conllevado a tomar medidas de urgencia en la educación universitaria, abordándose la modalidad virtual de modo obligatorio como opción para no paralizar el proceso formativo de los estudiantes. En tal sentido, se hace evidente el uso de teléfonos inteligentes, así como otros dispositivos tecnológicos como herramientas mediadoras en el proceso de aprendizaje, complementado por el uso personal-social que los estudiantes brindan a dichos dispositivos (Garrote-Rojas et al. 2018).

En complemento, la investigación de (Seong-Soo y Bo-Kyung, 2018) considera un factor adictivo la cantidad de tiempo invertido en redes sociales, juegos móviles, lo cual va en detrimento del rendimiento académico, constituyéndose en un factor de riesgo para alcanzar un efectivo aprendizaje en una sociedad cada vez más competitiva, mientras que si el uso es desarrollado con fines investigativos, de interacción con pares académicos, gira hacia un factor protector del crecimiento personal (Zwettler et al. 2018). Es también por estas razones que las personas adictas a los teléfonos inteligentes presentan problemas psicosociales en sus relaciones interpersonales, especialmente las relacionadas con la angustia y con la ansiedad tecnológica (Hyseni-Duraku y Hoxha, 2018; Rosen et al. 2018).

En conformidad con lo planteado, es pertinente estudiar la dependencia y adicción que muestran los estudiantes universitarios hacia el uso del teléfonos inteligentes, a partir de la aplicación de la EDAS (ArandaLópez et al. 2017), lo cual permite ahondar en conductas adictivas o problemáticas, con la finalidad de contribuir en la profundización de la literatura científica sobre el tema, específicamente, en población de habla hispana, distinguiéndose que el uso de dispositivos móviles hoy día es obligatorio en la vida social de las personas. Sin embargo, la diferencia se marca en que la persona adicta lo usa para drenar su disforia o malestar emocional, lo cual podría ir en detrimento de su salud; por tal motivo, la actual investigación a través de los resultados obtenidos, podrá alertar al establecimiento de estrategias o políticas educativas, en donde se aborde la salud de los estudiantes a partir de la adicción a teléfonos inteligentes como dispositivo de apoyo en la educación. Por consiguiente, el trabajo investigativo, tiene por objetivo determinar la dependencia y adicción a teléfonos inteligentes en estudiantes universitarios a partir de la aplicación de la EDAS.

\section{MÉTODO}

La metodología se presenta a través de seis subsecciones (S_1, S_2, S_3, S_4, S_5, S_6), subsecciones presentadas a continuación: contexto espacial geográfico de la investigación, diseño de investigación, descripción del instrumento de recopilación de datos, muestra poblacional, análisis estadístico, ética de la investigación. Con contexto nos referimos al ámbito espacial y geográfico donde se desarrolló la investigación. Con diseño hacemos referencia del propio diseño de investigación. Con descripción indicamos las características del instrumento empleado para la recolección de los datos. Con muestra poblacional hacemos referencia de la caracterización de las personas participantes en el llenado del instrumento de recolección de datos. Con análisis estadístico se indica el procedimiento empleado para la obtención de los resultados. Con ética nos referimos a los aspectos éticos propuestos por la ciencia con la finalidad de preservar la integridad de las personas e instituciones participantes como la veracidad de los datos analizados.

\section{S_1 Contexto espacial geográfico de la investigación}

La investigación, la cual se desarrolla en contexto universitario latinoamericano, es representada por cuatro países: Venezuela, Colombia, Ecuador y Perú, la intención de la misma consiste en dar a conocer el grado de adicción al teléfono inteligente en estudiantes de universidades en edades comprendidas entre los 18 y 31 años de edad, por cuanto a raíz de la pandemia por SARS-CoV-2, ha sido obligatorio la educación virtual en todos los niveles educativos, el uso de dispositivos móviles como recurso educativo. La recopilación de datos se desarrolló en cuatro universidades, localizadas en distintos países latinoamericanos: una Universidad Privada de Lima, Perú, una Universidad Privada de Colombia, una Universidad Pública de Venezuela, una Universidad Pública del Ecuador. 


\section{S_2 Diseño de investigación}

Se trabajó en función del método deductivo por cuanto se partió del razonamiento de los investigadores, el cual sería, verificado a través de los resultados de la investigación, mediante un tipo de investigación descriptiva con diseño de campo no experimental transversal, recopilándose la información en único momento investigativo a través de encuesta online y aplicación de EDAS, validada por (Aranda-López et al. 2017).

\section{S_3 Descripción del instrumento de recopilación de datos}

Se trabajó con EDAS, la cual consta de 40 ítems. La puntuación se registra de 1 a 5 , de menor a mayor intensidad, divididos en tres componentes: Componente 1, empleado para medir el uso, abuso y adicción a los teléfonos inteligentes y sus aplicaciones, el cual consta de 30 ítems (Item1, Item2, Item3, Item4, Item6, Item8, Item9, Item10, Item11, Item12, Item13, Item14, Item15, Item16, Item17, Item18, Item20, Item21, Item22, Item23, Item24, Item25, Item26, Item29, Item30, Item31, Item35, Item38, Item39, Item40); teniendo la siguiente escala: sin dependencia: $\leq 2,23$; dependencia: entre 2,24 hasta 3,5; adicción: $\geq 3,06$. Componente 2, empleado para detectar rasgos de personalidad, conformado por 6 ítems (Item5, Item7, Item19, Item27, Item33, Item36); enfocada primordialmente en medir (introversión-extroversión), la cual se relaciona con la estima de la persona y se tiene en cuenta: bajo: $\leq 2,23$; medio: entre 2,24 hasta 3,5 ; alto: $\geq 3,06$. Componente 3 , permite detectar el gasto monetario en aplicaciones y juegos móviles, constituido por 4 ítems (Item28, Item32, Item34, Item37); mide el gasto o inversión, a partir de la siguiente escala: bajo: $\leq 2,23$; medio: entre 2,24 hasta 3,5; alto: $\geq 3,06$. Siendo su puntuación mínima 1 y máxima 5.

\section{S_4 Muestra poblacional}

Se aplicó un muestreo estratificado proporcional a una muestra poblacional conformada por 303 estudiantes universitarios, distribuidos en 138 mujeres y 165 hombres, en un rango de edad comprendido entre los 18 y 31 años de edad, siendo la edad promedio constituida en 24,5 años, lo cual evidencia una población joven en formación universitaria. Dentro de los criterios de inclusión se consideraron los siguientes: ser mayor de edad, ser estudiante universitario, estar activos en el cursado del semestre a través de la modalidad educativa virtual. La muestra poblacional se estratifica en: 76 estudiantes de medicina, pertenecientes a una Universidad Pública de Venezuela; 75 estudiantes de medicina, cursando estudios en una Universidad Pública de Ecuador; 75 estudiantes de derecho, quienes cursan estudios de derecho en una Universidad Privada de Colombia; 77 estudiantes de administración de empresas, administración de negocios internacionales y contabilidad, los cuales cursan estudios en una Universidad Privada de Lima, Perú.

\section{S_5 Análisis estadístico}

Los datos, una vez recopilados, fueron organizados en la base de datos del programa Microsoft Excel 2016, luego fueron trasladados al programa IBM SPSS Statistics V25, procediéndose a calcular medias para los tres componentes de la EDAS, tal como se especifica en la misma para conocer el grado de adicción; complementándose con el cálculo de medias por género, edad y país.

\section{S_6 Ética de la investigación}

Se declara que no existe conflicto de interés entre los investigadores e instituciones, destacándose que la muestra poblacional participó libremente sin recibir retribución monetaria o no, siendo mayores de edad, manteniéndose confidencialidad del nombre de sujetos y universidades, por lo tanto, los datos recopilados son usados exclusivamente para fines investigativos, no experimentándose con humanos o animales durante el proceso.

\section{RESULTADOS}

En correspondencia a los datos recopilados y analizados, se presentan los resultados de la investigación. Para la interpretación del componente 1 . Se deben tener en cuenta los criterios planteados por (ArandaLópez, et al., 2017), quienes indican: sin dependencia: $\leq 2,23$; dependencia: entre 2,24 hasta 3,5; adicción: $\geq$ 3,06 . Por consiguiente, la media general fue de 3,87 , lo cual indica que existe dependencia y adicción en la muestra estudiada. En cuanto a la distribución porcentual, se tiene que el $72 \%$ de la muestra se encuentra en fase de adicción y 33\% en dependencia. El componente 2 en palabras de (Aranda-López, et al., 2017) mide primordialmente (introversión-extroversión). En mayor grado se identifica con una autoestima concatenada a la percepción de otros sujetos, siendo calificada del siguiente modo: bajo: $\leq 2,23$; medio: entre 2,24 hasta 3,5; alto: $\geq 3,06$. Como resultado, se obtuvo 3,89 como media general, lo cual evidencia que los sujetos estudiados presentan un alto grado de extroversión en su personalidad. En razón a la distribución porcentual, se tiene que el $73 \%$ de la muestra se encuentra en el rango de extroversión y $32 \%$ en introversión. 
Tabla 1: Componente 1. Uso, abuso y adicción al teléfono inteligente y sus aplicaciones.

\begin{tabular}{|c|c|}
\hline Item & Media \\
\hline I_1 & 3,90 \\
\hline I_2 & 3,88 \\
\hline I_3 & 3,92 \\
\hline I_4 & 3,92 \\
\hline I_5 & 3,90 \\
\hline I_6 & 3,80 \\
\hline I_7 & 3,90 \\
\hline I_8 & 3,80 \\
\hline I_9 & 3,92 \\
\hline I_10 & 3,80 \\
\hline I_11 & 3,90 \\
\hline I_12 & 3,92 \\
\hline I_13 & 3,80 \\
\hline I_14 & 3,92 \\
\hline I_15 & 3,63 \\
\hline I_16 & 3,92 \\
\hline I_17 & 3,92 \\
\hline I_18 & 3,80 \\
\hline I_19 & 3,92 \\
\hline I_20 & 3,90 \\
\hline I_21 & 3,92 \\
\hline I_22 & 3,92 \\
\hline I_23 & 3,80 \\
\hline I_24 & 3,92 \\
\hline I_25 & 3,80 \\
\hline I_26 & 3,90 \\
\hline I_27 & 3,92 \\
\hline I_28 & 3,80 \\
\hline I_29 & 3,90 \\
\hline I_30 & 3,80 \\
\hline N303 & Media general \\
\hline & $=3,87$ \\
\hline
\end{tabular}

Tabla 2: Componente 2. Rasgos de personalidad.

\begin{tabular}{|l|r|}
\hline \multicolumn{1}{|c|}{ Ítem } & \multicolumn{1}{|c|}{ Media } \\
\hline I_5 & 3,92 \\
\hline I_7 & 3,90 \\
\hline I_19 & 3,92 \\
\hline I_27 & 3,80 \\
\hline I_33 & 3,90 \\
\hline I_36 & 3,92 \\
\hline N303 & Media general $=3,89$ \\
\hline
\end{tabular}

Tabla 3: Componente 3. Gasto monetario en aplicaciones y juegos móviles.

\begin{tabular}{|l|r|}
\hline \multicolumn{1}{|c|}{ Ítem } & \multicolumn{1}{|c|}{ Media } \\
\hline I_28 & 3,80 \\
\hline I_32 & 3,92 \\
\hline I_34 & 3,80 \\
\hline I_37 & 3,92 \\
\hline N303 & Media general $=3,86$ \\
\hline
\end{tabular}

El componente 3 proyecta el gasto o inversión que realizan los usuarios en aplicaciones, juegos móviles, teléfonos inteligentes, (Aranda-López et al. 2017) esto implica que a mayor puntuación se genera mayor gasto o inversión, siendo medida del siguiente modo: bajo: $\leq 2,23$; medio: entre 2,24 hasta 3,5; alto: $\geq 3,06$. Al respecto, como resultado se evidenció una media general de 3,86 que denota un alto gasto o inversión por parte de los sujetos estudiados. En cuanto a la distribución porcentual, se tiene que el $72 \%$ de la muestra se genera altos gastos inversión y 33\% en moderada inversión. Se indica que la población femenina a pesar de ser menor en cantidad, representa una media por encima de la población masculina, denotándose así, mayor adicción al uso del teléfono inteligente. 
Tabla 4: Adicción al teléfono inteligente por género.

\begin{tabular}{|l|c|}
\hline \multicolumn{1}{|c|}{ Género } & Media general \\
\hline Femenino & 4,03 \\
\hline $\mathrm{N}$ & 138 \\
\hline Masculino & 3,73 \\
\hline $\mathrm{N}$ & 165 \\
\hline
\end{tabular}

Tabla 5: Adicción al teléfono inteligente por edad.

\begin{tabular}{|l|c|c|}
\hline \multicolumn{1}{|c|}{ Edad } & Media & $N$ \\
\hline 18 & 3,8 & 58 \\
\hline 19 & 4 & 36 \\
\hline 20 & 3,7 & 18 \\
\hline 21 & 4,3 & 24 \\
\hline 22 & 3,9 & 9 \\
\hline 23 & 3,7 & 18 \\
\hline 24 & 3,6 & 39 \\
\hline 25 & 3,8 & 23 \\
\hline 26 & 4 & 12 \\
\hline 27 & 3,7 & 15 \\
\hline 28 & 4,3 & 18 \\
\hline 29 & 4,1 & 14 \\
\hline 30 & 5 & 9 \\
\hline 31 & 3 & 10 \\
\hline Total Media & 3,9 & 303 \\
\hline
\end{tabular}

Se destaca que la población por edad en su totalidad, se encuentra en un promedio calificado como adicción al teléfono inteligente, sin embargo, la puntuación de mayor alcance se encuentra en los rangos de 21 y 28 años de edad con 4,3 de media, así como en el rango de 30 años de edad con 5 de media, lo cual implica que no existe una edad predeterminada u homogénea para padecer mayor o menor adicción. El país con mayor adicción a los dispositivos móviles, lo representa Colombia, aunque el resto se encuentra en el promedio determinado para ser considerados como adictos, lo cual representa la necesidad de ampliar en futuras investigaciones, las causas que lo ocasionan, como por ejemplo el nivel socioeconómico de vida.

Tabla 6: Adicción al teléfono inteligente por país.

\begin{tabular}{|l|c|c|}
\hline \multicolumn{1}{|c|}{ País } & Media & $N$ \\
\hline Venezuela & 3,79 & 76 \\
\hline Colombia & 4,02 & 75 \\
\hline Ecuador & 3,71 & 75 \\
\hline Perú & 3,87 & 77 \\
\hline Total media & 3,85 & 303 \\
\hline
\end{tabular}

\section{DISCUSIÓN}

El componente 1 de la EDAS, empleado para medir el uso, abuso y adicción al teléfono inteligente y sus aplicaciones, muestra en cada uno de sus 30 ítems, un resultado superior a $\geq 3,06$ siendo la media general de 3,87 distinguiéndose de ese modo, adicción en los usuarios encuestados. En tal sentido, se debe tener en cuenta que la adicción representa una afección para la salud integral de la persona, sin embargo, a juicio de Panova y Carbonell (2018), cuando se habla de comportamientos tecnológicos, no debe emplearse la palabra "adicción", sino, "uso problemático" o excesivo, esto involucra una controversia que debe ser develada con mayor profundización de estudios científicos en el área, postura compartida por Yu y Sussman (2020), al explicar la necesidad de mayores investigaciones para confirmar las actividades adictivas producto del uso de los dispositivos móviles.

A pesar de la controversia generada por Panova y Carbonell (2018); Yu y Sussman (2020), los resultados del componente 1, encuentran firme respaldo en la postura de Demirci et al. (2015), al indicar que la depresión, la ansiedad y la calidad del sueño pueden estar asociadas con el uso excesivo del teléfono inteligente, 
recomendando, además, hacer seguimiento a los estudiantes con la finalidad de confirmar la adicción a los teléfonos inteligentes. Esta visión es respalda por la investigación de Twenge (2018), quién presentó resultados donde se demuestra que, a menor tiempo dedicado a la comunicación electrónica, los usuarios eran más felices en comparación con quienes invertían mayor cantidad de tiempo al uso dispositivos móviles, siendo considerándose como un factor esencial de la disminución del bienestar de los adolescentes involucrados en el estudio.

Es de destacar, los resultados de Jasso-Medrano (2018), quien evidencia adicción a partir del uso de internet teléfono inteligente, lo cual abre el compás para comprender que por sí solo el dispositivo inteligente no genera adicción o uso excesivo, sino, en acompañamiento de factores como redes sociales, comunicación con amigos, uso de aplicaciones, tal como lo complementa lyengar, et al. (2020), quienes subrayan el uso primordial de este en tiempos de pandemia por COVID-19, proyectándose mayor utilidad a partir de la telemedicina, teleeducación, como espacios de auto desarrollo personal - social.

En otro orden de ideas, los resultados referidos al Componente 2, y se miden los rasgos de la personalidad, especialmente los referidos a introversión-extroversión, se indican resultados por encima de $\geq 3,06$ en cada uno de los ítems involucrados, resultando la media general de 3,89. Aparecen presencia de extroversión en la muestra estudiada, por ello, es considerable tener en cuenta los resultados de la investigación de Hong, (2020), quien afirma que cuanto más altas son las puntuaciones de extroversión y conciencia, mayor es el nivel de autoestima, capital psicológico y creatividad, lo cual permite en la persona, contar con un mejor manejo de la inteligencia emocional en favor de superar frustraciones o sentimiento negativos que podrían desencadenar en ansiedad, depresión y estrés Martínez (2021). En contraparte, el estudio de Kwon y Choi (2021), ratifica que, aunque los estudiantes universitarios tenían una orientación extrovertida, no tuvo una influencia significativa en las emociones positivas cuando su nivel de evitación del apego era alto, en consideración cuando este no es manejado asertivamente se originan afectos no operativos en detrimento de la vida emocional de la persona.

Por otra parte, los resultados del componente 3 , empleado para medir el gasto monetario en aplicaciones y juegos móviles, fue mayor a $\geq 3,06$ en cada ítem involucrado, siendo la media general de 3,86 , lo cual evidencia un alto gasto por parte de la muestra poblacional. Esta acción es respalda por la investigación de Berthon (2019), al indicar que dormimos con estos dispositivos, trabajamos y jugamos con ellos, consumiendo cada vez más nuestro tiempo, atención y dinero, de este modo, nos constituimos en adictos al dispositivo móvil o quizás a las prácticas interactivas generadas y, por tal razón, adoptamos conductas que proyectan ser cada día más adictivas. Al respecto, Corzo et al. (2020), explican en su estudio, la existencia de políticas comerciales para el fortalecimiento la triada cliente-industria-gobierno, con fines de promover mayor competitividad, repercutiendo en las necesidades psicoemocionales de las personas mediante el uso de un teléfono inteligente de mayor espectro que permite acceder al mundo digital y ha sido reforzado en la nueva normalidad social producto de la pandemia por COVID-19.

En cambio, los resultados de la actual investigación presentan mayor adicción en la población femenina estudiada, siendo confirmatorio del estudio de García-Domingo et al. (2020), donde se revela que las mujeres pasan más tiempo usando el teléfono inteligente. Por su parte, Chen (2017), confirma la conducta adictiva por parte de estudiantes universitarios al uso del teléfono inteligente, pero destaca que no existe una asociación directa con el sexo, recomendando, además, involucrar estrategias para minimizar la adicción en los estudiantes de pregrado. Vale resaltar que la investigación de Chen es de 2017, en donde no existía confinamiento y distanciamiento social a causa de la pandemia por COVID-19, lo cual permite inferir que la adicción seguirá en crecimiento en los próximos tiempos, siendo este el factor primordial que debe ser abordado para evitar problemas en la salud de los usuarios, sin distinción de sexo. Este último elemento constituye un punto referencial para próximas investigaciones donde se profundice la asociación del sexo con el nivel de adicción al dispositivo móvil.

En relación a los resultados por edad y país, la investigación de Christensen (2016), confirma que los adultos pasan una cantidad sustancial de tiempo usando sus teléfonos inteligentes, siendo similar a los actuales resultados donde la muestra poblacional de 28 y 30 años de edad presenta la puntuación más alta, con 4,3 y 5 de media respectivamente, excediendo el rango de $\geq 3,06$. En cuanto al país, el uso difiere según la edad y la raza, pero es similar entre los estratos socioeconómicos, lo que sugiere que los factores culturales pueden impulsar el uso de teléfonos inteligentes e implica que una población universitaria con acceso a financiamiento económico usa el teléfono inteligente como un dispositivo indispensable. En virtud de ello, se considera parte de la cultura de la generación digital y se acrecienta la posibilidad de sufrir adicción, tal como lo indican los resultados de la actual investigación.

La puntuación alcanzada en los componentes 1,2 y 3, dan como resultado una conducta adictiva, lo cual, debe conllevar a reflexionar el uso que se le brinda al teléfono inteligente, así como reconducir la utilización de herramientas basadas en TIC y redes sociales como parte del crecimiento personal, social y educativo 
Garrote-Rojas et al. (2018). Paradójicamente, al problema de adicción al dispositivo móvil, Pórarinsdóttir, (2017) y Higgins (2016), invitan a usarlo como una herramienta a través de la cual, se pueden generar aplicaciones para el cuidado de la salud y prevención del estrés. Tal aseveración es compatible con lo planteado por Garrote-Rojas, et al. (2018), al destacar la necesidad de ajustarlas al uso del teléfono inteligente, por cuanto Elhai et al. (2020), confirman que la generación de ansiedad y depresión se producen por abuso en el empleo de estos dispositivos en estudiantes universitarios, considerándose como factores predictores de problemas psicosociales en la relación interpersonal, tal como aseveran Seong-Soo y BoKyung (2018), siendo fundamental alertar a la sociedad sobre los riesgos que se corre al usarlos excesivamente en actividades no académicas o afines a la investigación científica, es decir, dándole mayor proporción a juegos u afines, los cuales a su vez, contribuyen en profundizar la dependencia - adicción, sobre todo si el individuo con problemas de estima, percibe como un recurso fundamental el dispositivo móvil, para establecer vínculos afectivos o de apego con sus pares sociales.

Siendo considerable, tener en cuenta la investigación de Hyseni-Duraku y Hoxha (2018), la cual demuestra la existencia de una asociación significativa entre angustia psicológica y la autoestima con el éxito académico, es decir, a mayor o mejor estima en el estudiante, se demostró mayor proporción en padecer estrés por lograr un rendimiento alto en sus estudios, esto puede influir en el uso del teléfono inteligente como elemento generador de dependencia y adicción, reforzado por el período obligatorio de emplear la modalidad virtual como parte de la nueva normalidad social a causa del COVID-19, siendo concomitante con los resultados obtenidos para el componente 2 en la actual investigación, por cuanto esta mide rasgos de personalidad como introversión - extroversión, siendo esta última asociada a una persona con auto concepto significativo de sí mismo, siendo que su identidad social se convierte en un factor protector de su salud mental Zwettler et al. (2018), indicios que deben ser tenido en cuenta por las autoridades universitarias con la finalidad de generar estrategias de abordaje preventivo - clínico de los estudiantes, a través de los departamentos de asistencia estudiantil, los cuales deben ser reconducidos para abordar afecciones derivadas de la interrelación fundamentada en el uso adictivo del dispositivo móvil, entre las cuales se encuentra la ansiedad tecnológica como factor de riesgo para una adecuada metacognición por parte del estudiante universitario (Rosen et al. 2018).

Los autores de la actual investigación, concuerdan con los investigadores que consideran el uso excesivo del teléfono inteligente como una adicción sin sustancia, sin embargo, se deja abierto el debate a partir de la postura de (Panova y Carbonell, 2018; Yu y Sussman, 2020), sobre reconducir el término para referirse a este tipo de conducta, más aún, cuando (Carlisle, 2020), en su investigación destaca que la Asociación Estadounidense de Psiquiatría (APA), ha solicitado que se realicen más investigaciones sobre el trastorno de los juegos de Internet (IGD) colocándolo en la Sección III del Manual de diagnóstico y estadísticas (DSM-5 ). Todo ello, guarda relación con el tema de adicción al teléfono inteligente, posibilitándose la generación de un mayor número de trabajos investigativos con la intención de aclarar desde una óptica científica, la terminología adecuada, así como los factores intervinientes en el uso del dispositivo móvil como proceso adictivo en la población universitaria.

\section{CONCLUSIONES}

Una vez descritos los resultados y discusión de la investigación, a través de la confrontación teórica de la postura de diversos autores, se presentan las siguientes conclusiones: 1 . Una vez aplicada la EDAS, siendo procesados estadísticamente los datos recopilados, se determina que existe dependencia y adicción al teléfono inteligente en estudiantes universitarios, en razón de la muestra estudiada; 2. La población femenina estudiada presenta mayor dependencia y adicción en comparación a la muestra masculina; 3. Existe controversia entre autores que difieren del tema adicción, en su lugar emplean uso problemático o excesivo, lo cual abre un debate si realmente existe adicción o no hacia el teléfono inteligente; 4 . La mayor afección se encuentra relacionada a la ansiedad, la depresión, el estrés, los trastornos del sueño, como elementos generadores de dependencia y adicción al teléfono inteligente por parte de los estudiantes universitarios.

\section{REFERENCIAS}

American Psychiatric Association. Diagnostic and Statistical Manual of Mental Disorders, 5th. Edition (DSM-5), 316-316. American Psychiatric Association, Washington, USA (2013)

Aranda, M., Fuentes, V., y García-Domingo, M., No sin mi Smartphone. Elaboración y validación de la Escala de Dependencia y Adicción al Smartphone (EDAS), http://dx.doi.org/10.4067/S0718-48082017000100004, Ter. Psicol., 35(1), 35-45 (2017)

Berthon, P., Pitt, L., y Campbell, C., Addictive De-Vices: A Public Policy Analysis of Sources and Solutions to Digital Addiction, https://doi.org/10.1177/0743915619859852, Journal of Public Policy \& Marketing, 38(4), 451-468. (2019)

Carlisle, K., Utility of DSM-5 Criteria for Internet Gaming Disorder, https://doi.org/10.1177/0033294120965476,

Psychological Reports, (2020) 
Chen, B., Liu, F., y otros 4 autores, Gender differences in factors associated with smartphone addiction: a crosssectional study among medical college students, https://doi.org/10.1186/s12888-017-1503-z, BMC psychiatry, 17(1), 341 (2017)

Christensen, M. A., Bettencourt, L., y otros 6 autores, Direct Measurements of Smartphone Screen-Time: Relationships with Demographics and Sleep, https://doi.org/10.1371/journal.pone.0165331, PloS one, 11(11), e0165331 (2016)

Corzo, G., y Álvarez-Aros, E., Estrategias de competitividad tecnológica en la conectividad móvil y las comunicaciones de la industria 4.0 en Latinoamérica, https://dx.doi.org/10.4067/S0718-07642020000600183. Inf. Tecnol., 31(6), 183-192 (2020)

Demirci, K., Akgönül, M., y Akpinar, A., Relationship of smartphone use severity with sleep quality, depression, and anxiety in university students, https://doi.org/10.1556/2006.4.2015.010, Journal of behavioral addictions, 4(2), 85-92 (2015)

Elhai, J., Rozgonjuk, D., y otros 6 autores, Excessive reassurance seeking mediates relations between rumination and problematic smartphone use, https://doi.org/10.1521/bumc_2020_84_07, Bulletin of the Menninger Clinic, 84(2), 137-155 (2020)

García-Domingo, M., Fuentes, V., Pérez-Padilla, J., y Aranda, M., EDAS-18: Validación de la versión corta de la escala de dependencia y adicción al Smartphone, https://doi.org/10.4067/S0718-48082020000300339, Ter. Psicol., 38(3), 339 $361(2020)$

Garrote-Rojas, D., Jiménez-Fernández, S., y Gómez-Barreto, I., Problemas Derivados del Uso de Internet y el Teléfono Móvil en Estudiantes Universitarios, https://dx.doi.org/10.4067/S0718-50062018000200099, Form. univ., 11(2), 99-108 (2018)

Higgins J. P., Smartphone Applications for Patients' Health and Fitness, https://doi.org/10.1016/j.amjmed.2015.05.038, American journal of medicine, 129(1), 11-19 (2016)

Hong, M., Dyakov, D., y Zheng, J., Self-esteem and psychological capital: Their mediation of the relationship between Big Five personality traits and creativity in college students, https://doi.org/10.1080/14330237.2020.1744286, Journal of Psychology in Africa, 30(2), 119-124 (2020)

Hyseni-Duraku, Z., y Hoxha, L., Self-esteem, study skills, self-concept, social support, psychological distress, and coping mechanism effects on test anxiety and academic performance, https://doi.org/10.1177/2055102918799963, Health psychology open, 5(2), 1-19 (2018)

lyengar, K., Upadhyaya, G. K., Vaishya, R., y Jain, V., COVID-19 and applications of smartphone technology in the current pandemic, https://doi.org/10.1016/j.dsx.2020.05.033, Diabetes \& metabolic syndrome, 14(5), 733-737 (2020)

Jasso-Medrano, J., López-Rosales, F., y Díaz-Loving, R., Conducta adictiva a las redes sociales y su relación con el uso problemático del móvil, https://doi.org/10.1016/j.aipprr.2017.11.00, Acta De Investigación Psicológica, 7(3), 2833-2838 (2018)

Kwon, C., y Choi, E., Extroversion and Subjective Well-Being Among Korean University Students: The Moderating Role of Attachment, https://doi.org/10.1177/0033294121991828, Psychological Reports. (2021).

Martínez, A., Inteligencia emocional en el talento humano empresarial, Revista Multidisciplinaria Perspectivas Investigativas, ISSN: 2773-7411, 1(1), 23-29, (2021)

Panova, T., y Carbonell, X., Is smartphone addiction really an addiction?, https://doi.org/10.1556/2006.7.2018.49, Journal of behavioral addictions, 7(2), 252-259 (2018)

Rosen, L., Carrier, M., y otros siete autores; The Role of Executive Functioning and Technological Anxiety (FOMO) in College Course Performance as Mediated by Technology Usage and Multitasking Habits, https://doi.org/10.5093/psed2018a3, Psicología Educativa, 24(1), 14-25 (2018)

Seong-Soo C., y Bo-Kyung S., Smartphone use and smartphone addiction in middle school students in Korea: Prevalence, social networking service, and game use, https://doi.org/10.1177/2055102918755046, Health psychology open, 5(1), 1-15 (2018)

Pórarinsdóttir, H., Kessing, L. V., y Faurholt-Jepsen, M., Smartphone-Based Self-Assessment of Stress in Healthy Adult Individuals: A Systematic Review, https://doi.org/10.2196/jmir.6397, Journal of medical Internet research, 19(2), e41 (2017)

Twenge, J. M., Martin, G. N., y Campbell, W. K., Decreases in psychological well-being among American adolescents after 2012 and links to screen time during the rise of smartphone technology, https://doi.org/10.1037/emo0000403, Emotion, 18(6), 765-780 (2018)

Yu, S., y Sussman, S., Does Smartphone Addiction Fall on a Continuum of Addictive Behaviors?, https://doi.org/10.3390/ijerph17020422, International journal of environmental research and public health, 17(2), 422-442 (2020)

Zwettler, C., Reiss, N., y otros 4 autores, The relation between social identity and test anxiety in university students, https://doi.org/10.1177/2055102918785415, Health psychology open, 5(2), 1-7 (2018) 